Research Article

\title{
Pressure Dependence of the Electrical Resistivity in Polymer Polyaniline
}

\author{
Daihui Huang, ${ }^{1}$ Dong Xie, ${ }^{1}$ Jingjing Gao, ${ }^{2}$ Wangchun Lv, ${ }^{3}$ and Shiming Hong ${ }^{1}$ \\ ${ }^{1}$ College of Physical Science and Technology, Southwest Jiaotong University, Chengdu 610031, China \\ ${ }^{2}$ College of Materials Science and Engineering, Sichuan University, Chengdu 610064, China \\ ${ }^{3}$ College of Materials Science, Southwest Jiaotong University, Chengdu 610031, China \\ Correspondence should be addressed to Daihui Huang; forever-2002@tom.com
}

Received 3 February 2013; Accepted 15 April 2013

Academic Editor: Zhimin Liu

Copyright (C) 2013 Daihui Huang et al. This is an open access article distributed under the Creative Commons Attribution License, which permits unrestricted use, distribution, and reproduction in any medium, provided the original work is properly cited.

\begin{abstract}
Polyaniline (PAN) was prepared by using a technique of chemical synthesis to obtain the insulating emeraldine base form. And then PAN was doped with toluenesulfonic acid (TSA), $\mathrm{HCl}$, or camphor sulfonic acid (CSA) to protonate it into conducting salt form. The morphologies and electrical property of PAN under atmospheric pressure were investigated. Subsequently, the high pressure using a Bridgman anvil cell was applied on the doped PAN, and the effect of high pressure on the properties of doped PAN was analyzed. At normal pressure, the conductivity of PAN increases as the PH value increases. While at high pressures, the conductivity of PAN increases, and then it becomes independent of pressure. The results indicate that the conductivity of PAN is related to the presence of the polaron band, and the doped PAN under high pressure will be enhanced strongly in conductivity because of overlap of polaron band and $\pi$ band. However, with the further increase of the applied pressure, scattering mechanisms of carriers limit the conductivity of PAN.
\end{abstract}

\section{Introduction}

PAN is one of the most widely investigated conducting polymers because of its diversified structure, excellent physical and chemical properties, good stability, and a unique doping mechanism [1-12], among which the electrical property of PAN has been paid the most attention.

The conductivity of PAN is not high enough $[1,4]$. To overcome this problem is to use the doping process $[6,9]$ or to apply the high pressure, while PAN under high pressure will show different electrical properties in contrast to the atmospheric pressure, which can be widely applied in fields such as new pressure calibration materials, buffer electrode materials, recharge batteries, and solar cells [13-24].

Generally, there are two methods to study the structure and properties of PAN under pressure. One is application of high pressures after the synthesis of PAN; the other is in situ polymerization of PAN under various pressures $[13,16]$. The latter, however, has some disadvantages; for example, the reactor must be maintained at low temperature $\left(5^{\circ} \mathrm{C}\right)$. In addition, the applied pressure is not high enough $\left(10^{8} \mathrm{pa}\right)$.
To investigate the structural and electro-optical properties of PAN synthesized under atmospheric pressure, the high pressure apparatuses such as the diamond anvil cell and Bridgman anvil cell are often used. It is difficult to obtain high pressure by using the hydrostatic high pressure apparatus [18], and the sample shape may change in the process of applying high pressure. Although the diamond anvil cell $[19,20]$ can reach a very high pressure ( $66 \mathrm{GPa}$ ), its pressure datum is very large compared with similar devices. The electrical properties of PAN with a certain dopant and a certain doping concentration were studied by Cui et al. [17] using the Bridgman anvil cell, but they did not interpret the saturation section in pressure-resistance curves.

The electrical property of PAN under high pressure changes greatly in contrast to the atmospheric pressure. Moreover, little work has been reported on PAN with different doping concentrations and doping agents under high pressure. In this work, we present the electrical resistivity measurements at high pressure using the Bridgman anvil cell on samples of PAN and analyze intensively the mechanism of conduction in PAN. 


\section{Experimental Details}

2.1. Synthesis of PAN. Aniline was distilled and purified under reduced pressure in the atmosphere of zinc powder. All other reagents were of analytical grade, and the solution was prepared by deionized water. PAN was synthesized using the chemical technique of Chiang and MacDiarmid [1] to obtain the insulating emeraldine base form. Aniline was needed, and ammonium sulfate was oxidizing agent. And then PAN was doped with TSA, $\mathrm{HCl}$, or CSA. Cresol was the solution. The experimental details are presented in [2].

2.2. Post Treatments of PAN. PAN was loaded into the mold and pressed into a diameter of $4 \mathrm{~mm}$ and the thick of $1 \mathrm{~mm}$ discs under a certain pressure. Two pyrophyllite gaskets (26 $\mathrm{mm}$ in diameter, $1.05 \mathrm{~mm}$ in thickness, with a hole of $4 \mathrm{~mm}$ ) were used as the pressure medium and sealer. PAN discs were fitted in the central holes of the pyrophyllites. Mica pieces of $0.05 \mathrm{~mm}$ thickness were placed on the top and the bottom of the samples as the insulators. Four copper wires (i.e., A, B, C, and D) were inserted between the two sample discs to perform the electrical resistance measurements by the four-probe Van der Pauw method. The schematic sample PAN assembly is shown in Figure 1. A set of Bridgman anvils [21] was used to produce high pressure. The anvil is made of tungsten carbide, and the top diameter is $26 \mathrm{~mm}$. The relationship between the oil pressure and the actual pressure in the sample was calibrated by using the known phase transitions of bismuth and barium. The compression in the sample was considered to be nearly hydrostatic because the size of the sample is sufficiently smaller than the compressible gaskets, and so it is in the elastic region of the soft medium gaskets.

\section{Results and Discussion}

Figure 2(a) shows the SEM (Japan JSM-5900LV) images of the sheet made from the doped PAN. The surface is relatively flat, and the distance between the PAN molecular chains is not reduced because of the low pressure. So, the properties of PAN are only related to the doping type and doping concentration, which are in agreement with the results of the electrical property under the atmospheric pressure. As shown in Figure 2(b), the surface of PAN sheet at high pressure $(\sim 2 \mathrm{GPa})$ becomes denser and rougher, which is the same as the morphology observed by Karami et al. [14] who did not find a network made of nanofibrils [16]. It is found that some regions have a clear network morphology, which is similar to the nanostructured PAN of 2D, 3D mesh prepared by the electrochemical method [25]. The formation of the network morphology is related to the reduced distance between the molecular chains, which leads to the decrease of the internal infects and free volume in materials; thus, the electrical property is significantly improved.

Table 1 shows that the resistivity of the doped PAN decreases significantly with the increase of doping concentration under the atmospheric pressure. The resistivity of PAN doped with various acids is different due to the unique conductivity mechanisms of PAN. When PAN is doped with different acids, the electronic structure changes a lot that
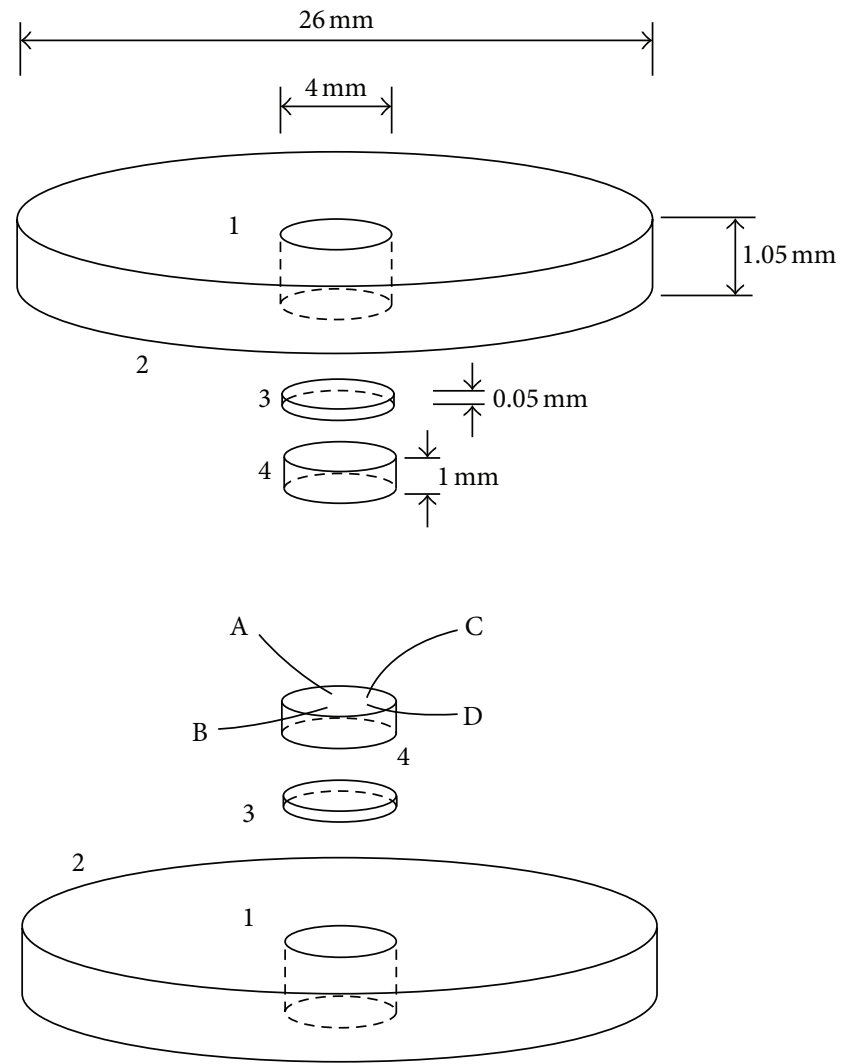

FIgURE 1: Sample assembly for measuring electrical resistivity of PAN. 1: hole, 2: pyrophyllite, 3: mica disc, 4: sample PAN, and A$\mathrm{D}: \mathrm{Cu}$ wire.

causes huge differences in electronic property, as confirmed by Raman spectroscopy or UV-vis-NIR spectra $[4,6,9]$. Cochet et al. [9] investigated the changes of electronic structure and vibration patterns of PAN doped with $\mathrm{HCl}$ and CSA by Raman spectroscopy and UV-vis-NIR spectra. Besides, Rannou et al. [6] found that different dopants mixing with different solvents can form a secondary doping [4], which makes a coillike conformation of PAN into an expanded coillike conformation and thereby significantly enhances the conductivity of PAN. In this work, the solvent is m-cresol; the electrical property of PAN treated with three kinds of acids is different. The resistivity for doped samples is close to the data reported in the literatures $[4,10]$.

The pressure dependence of the conductivity of doped PAN is shown in Figures 2-4. Figure 2 shows the effect of different concentrations of CSA on the PAN conductivity at high pressure. For the doped sample with the $0.05 \mathrm{M} \mathrm{CSA}$, the conductivity tends to reach saturation $(\sim 26 \Omega \mathrm{cm})$ under $3.5 \mathrm{GPa}$, while for the heavily doped $(0.1 \sim 0.5 \mathrm{M})$ sample, the conductivity tends to reach saturation $(\sim 2.5 \Omega \mathrm{cm})$ under $1.5 \mathrm{GPa}$. For the $\mathrm{HCl}$-doped sample (Figure 3 ), the conductivity almost does not change with increasing pressure more than $2.5 \mathrm{GPa}$ at the low concentrations, while the conductivity becomes independent of pressure under 1.0 GPa at the high concentrations. Similar behavior for PAN doped with $0.5 \mathrm{M}$ TSA is observed as shown in Figure 4. 


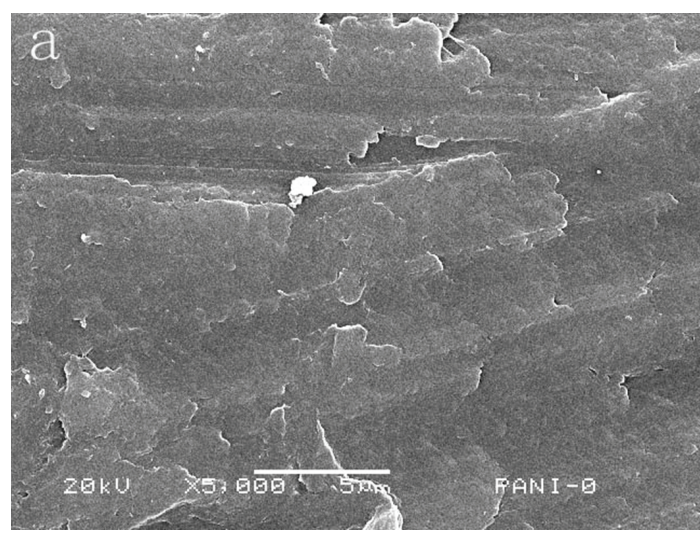

(a)

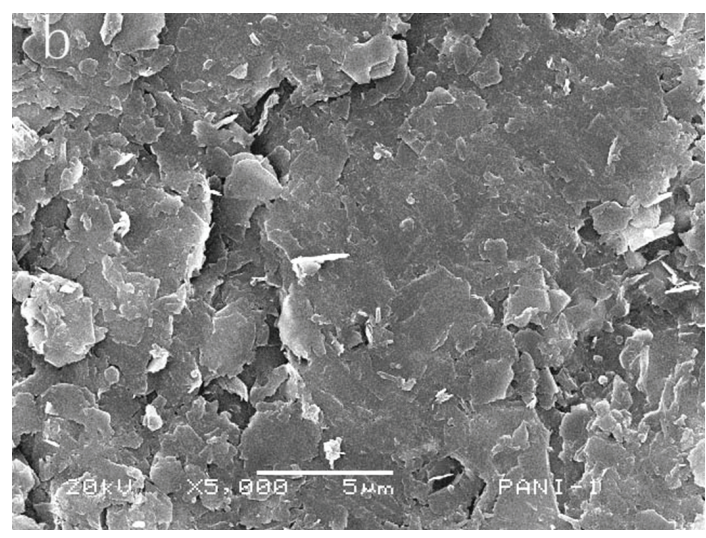

(b)

Figure 2: SEM of PAN (a) under atmospheric pressure and (b) at a pressure of $2.0 \times 10^{9} \mathrm{~Pa}$.

TABLE 1: Resistivity of doped PAN under atmospheric pressure.

\begin{tabular}{lccc}
\hline Doping type & $\begin{array}{c}\text { Doping } \\
\text { concentration }(\mathrm{M})\end{array}$ & \multicolumn{2}{c}{ Resistivity $(\Omega \mathrm{cm})$} \\
This work & Related work \\
\hline CSA & 0.05 & 50 & \\
CSA & 0.1 & 10 & $\sim 10[4]$ \\
CSA & 0.5 & 5 & \\
\hline $\mathrm{HCl}$ & 0.5 & 4.5 & $\sim 0.3[4,10]$ \\
$\mathrm{HCl}$ & 2 & 0.4 & \\
\hline TSA & 0.5 & 0.24 & \\
TSA & 1 & 0.032 & $\sim 0.01[4]$ \\
TSA & 2.5 & 0.041 & \\
\hline
\end{tabular}

With the increase of pressure, the electrical property of the doped PAN at low concentration changes significantly (Figures 3-5). The resistivity decreases rapidly, but when the pressure is up to a certain value $(1.0 \sim 3.5 \mathrm{GPa})$, the change in resistivity will tend to saturate, which is in good agreement with the result of the other reported literatures [14-20]. The reason is that when high pressure is applied, the distance of molecular chain decreases; the overlap of molecular orbit and the interactions of interchain increase; thus, the electrical conductivity of PAN increases. For PAN doped with TSA,

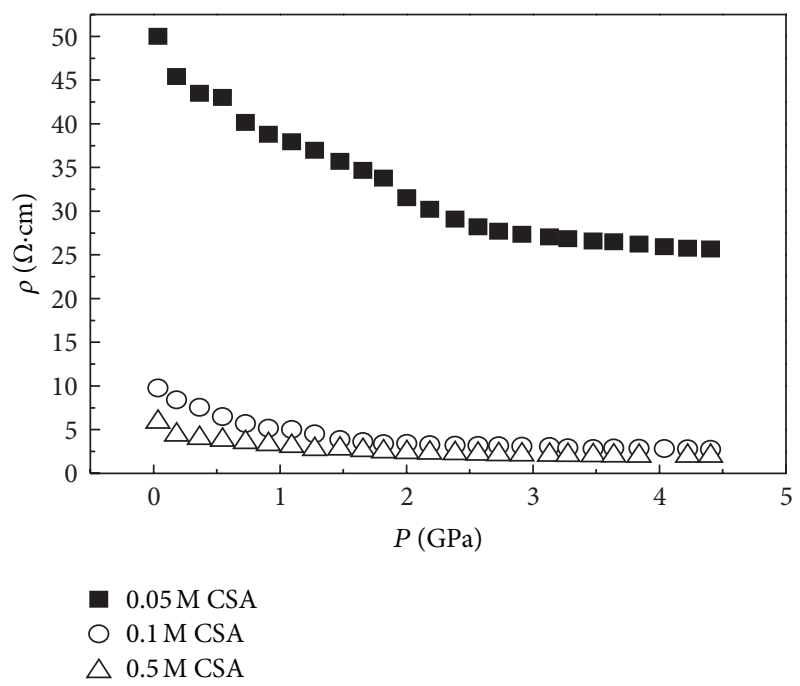

FIGURE 3: Effect of concentrations of CSA on resistivity of PAN under high pressure.

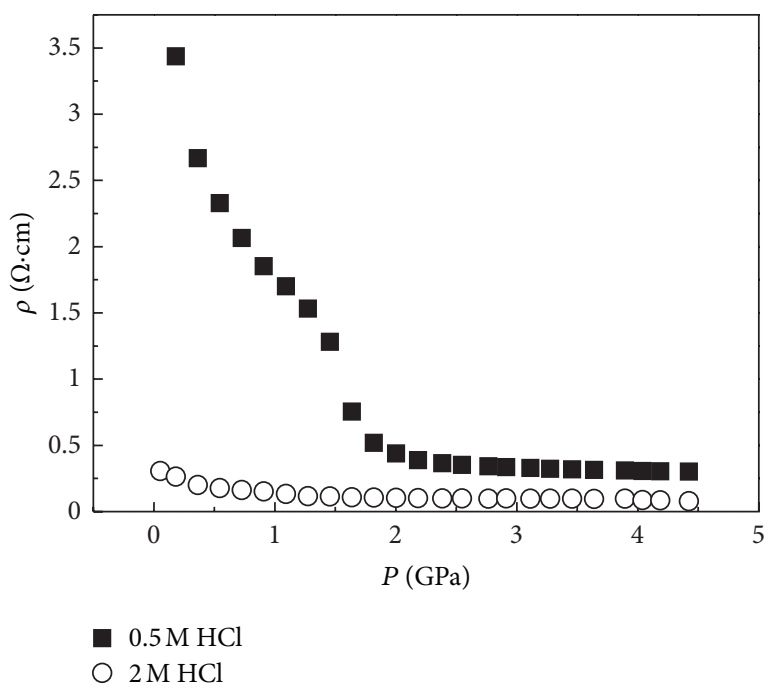

FIGURE 4: Effect of concentrations of $\mathrm{HCl}$ on resistivity of PAN under high pressure.

$\mathrm{HCl}$, or CSA, the changes in resistivity with increasing pressure are not very obvious at high doping concentration (Figures 3-5). Although the saturation regions of resistivity are different with various dopants, the resistivity of PAN as function of pressure shows the similar trend (Figures 3$5)$. The differences in saturation regions may be related to test methods [14, 15, 17-19], doping agents, or synthetic process [20]. For example, there are differences between the experimental and Hertz formula conditions at the highpressure experiments. Besides, the sensitivity of pressure is different for the various doping systems, which is mainly due to the different electronic structure for PAN after doping [9].

Now, we discuss the influences on electrical properties of PAN caused by doping acids and applying pressures according to energy band structures. The conductivity of solid is generally found to be correlated with carriers such as 


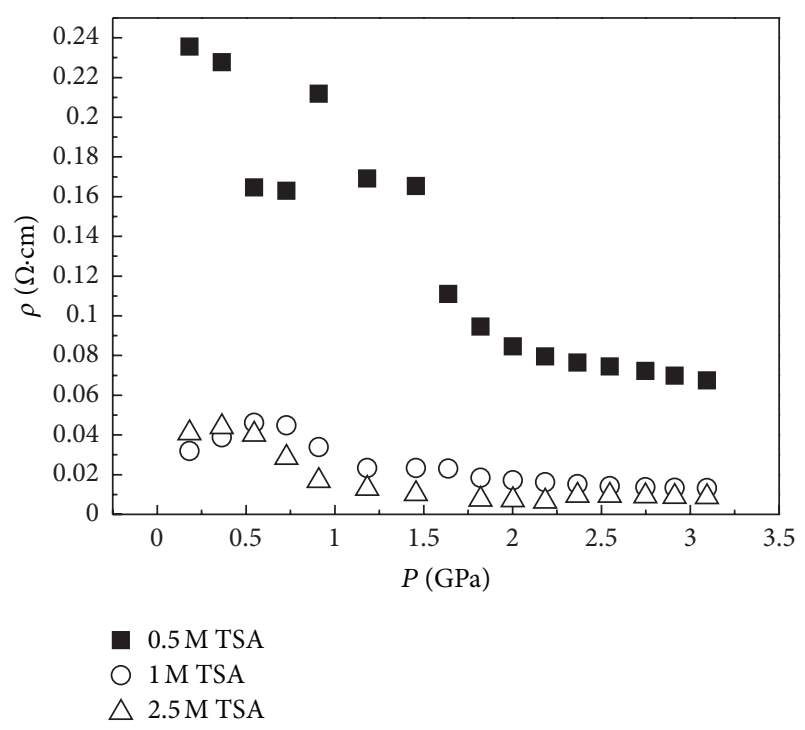

FIGURE 5: Effect of concentrations of TSA on resistivity of PAN under high pressure.

free electron, electron, or hole. The conducting mechanism of conducting polymer is different from the metal and semiconductor. The carriers of conducting polymer may be soliton, polaron, or dipole. As can be seen from the energy band structure (Figure 6), intrinsic PAN only shows optical transition: $\pi \rightarrow \pi^{*}$, which means that energy band gap is $3.44 \mathrm{eV}$. Therefore, intrinsic PAN is an insulator (Figure 6(a)).

When PAN is doped with TSA, $\mathrm{HCl}$, or CSA, it produces unstable dipole; then the dipole separates into the polaron that forms half-filled polaron band in the energy gap (Figure 6(b)) [26], whose site is $1.59 \mathrm{eV}$ away from $\pi$ band and about $2.82 \mathrm{eV}$ from $\pi^{*}$ band. It is clear that the carrier is easy to form transition of polaron band $\rightarrow \pi^{*}$ and $\pi \rightarrow$ polaron band at the presence of the polaron band, and the conductivity of PAN is enhanced.

When pressure is applied, the defects in PAN decrease. This leads to the decrease of possibility of polar generation and annihilation and the increase of interactions and delocalization. Meanwhile, the transition probability of carrier through the polaron band increases, so the conductivity of PAN increases. Molecular chains of PAN stretch fully, and conformations change with increasing the pressure, and the polaron bands disperse to a higher degree in the energy band structure and overlap with $\pi$ band. The transition of $\pi \rightarrow \pi^{*}$, polaron band $\rightarrow \pi^{*}$, and $\pi \rightarrow$ polaron band will disappear; instead, free carrier absorption will present similar to the metal (solid arrow in Figure 6(c)). Therefore, it shows the metal-like conductivity mechanism. The overlapping phenomena become more obvious as the pressure increases and the electrical conductivity will increase due to the increases of the carrier concentration (Figures 3-5). However, when the pressure reaches to a certain value (such as $1.0 \sim 3.5 \mathrm{GPa}$ in this work), the pressure saturation regions in the curves are present because the scattering mechanisms that limit the mobility of carriers with the increase of the carriers should be
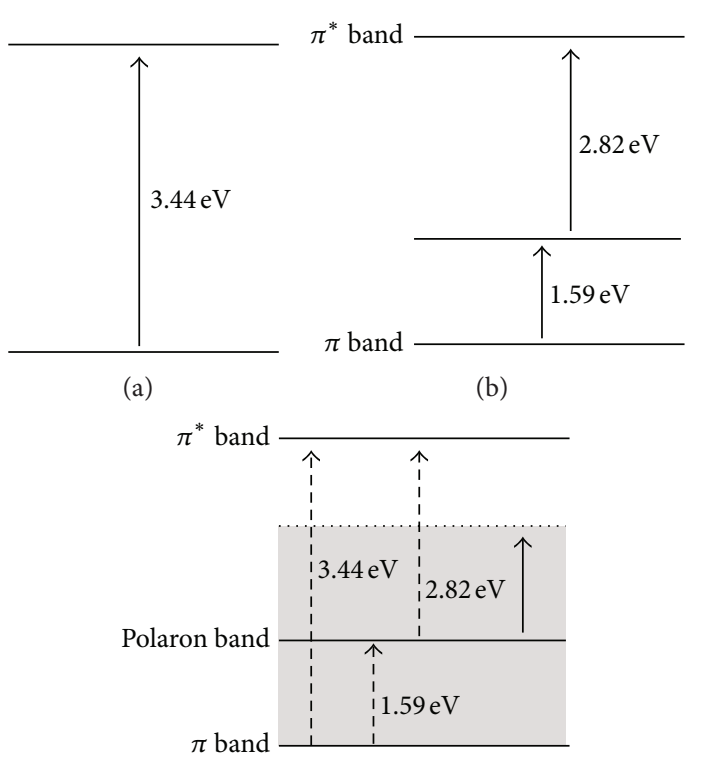

(c)

FIgURE 6: Energy band structure of (a) emeraldine base, (b) doped PAN, and (c) doped PAN under high pressure.

taken into account [27]. Finally, electrical resistivity of PAN reaches saturation (Figures 3-5).

\section{Conclusions}

PAN was prepared by using the technique of chemical synthesis. The electrical conductivity of PAN with different doping systems and doping concentrations could be improved in a certain extent under the atmospheric pressure, while it increases by an order of magnitude (as $0.5 \mathrm{M} \mathrm{HCl}$ doping: $4.5 \Omega \mathrm{cm} \rightarrow 0.3 \Omega \mathrm{cm}$ ) under high pressure. The increase of the PAN conductivity is mainly related to the dispersion effect of the polaron band and the increase of the carrier concentration under high pressure. The presence of the polaron band leads to the electrical conductivity change in PAN. When the applied pressure reaches to a certain value (1.0 3.5 GPa), the scattering mechanisms of carriers for PAN limit the increase of the electrical conductivity.

\section{Acknowledgment}

Financial support from the Fundamental Research Funds for the Central University (Grant no. SWJTU12CX087) and the National Natural Science Foundation of China (Grant nos. 61076058 and 81171462 ) is gratefully acknowledged.

\section{References}

[1] J. C. Chiang and A. G. MacDiarmid, “Polyaniline': protonic acid doping of the emeraldine form to the metallic regime," Synthetic Metals, vol. 13, no. 1-3, pp. 193-205, 1986.

[2] M. Reghu, Y. Cao, D. Moses, and A. J. Heeger, "Counterioninduced processibility of polyaniline: transport at the metalinsulator boundary," Physical Review B, vol. 47, no. 4, pp. 17581764, 1993. 
[3] R. Murugesan and E. Subramanian, "Effect of organic dopants on electrodeposition and characteristics of polyaniline under the varying influence of $\mathrm{H}_{2} \mathrm{SO}_{4}$ and $\mathrm{HClO}_{4}$ electrolyte media," Materials Chemistry and Physics, vol. 80, no. 3, pp. 731-739, 1993.

[4] A. G. MacDiarmid and A. J. Epstein, "Secondary doping in polyaniline," Synthetic Metals, vol. 69, no. 1-3, pp. 85-92, 1995.

[5] O. P. Dimitriev, "Origin of the exciton transition shift in thin films of polyaniline," Synthetic Metals, vol. 125, no. 3, pp. 359363, 2001

[6] P. Rannou, A. Pron, and M. Nechtschein, "UV-vis-NIR studies of new PANI/dopant/solvent associations with metallic-like behaviour," Synthetic Metals, vol. 101, no. 1, pp. 827-828, 1999.

[7] O. P. Dimitriev, "Doping of polyaniline by transition metal salts: effect of metal cation on the film morphology," Synthetic Metals, vol. 142, no. 1-3, pp. 299-303, 2004.

[8] P. N. Adams, S. J. Pomfret, and A. P. Monkman, "Conductivity measurements of novel, oriented polyaniline films," Synthetic Metals, vol. 101, no. 1, pp. 776-777, 1999.

[9] M. Cochet, B. Corraze, S. Quillard, J. P. Buisson, S. Lefrant, and G. Louarn, "Electronic and vibrational changes induced by different acidic vapors in polyaniline," Synthetic Metals, vol. 84, no. 1-3, pp. 757-758, 1997.

[10] D. Cottevieille, A. Le Méhauté, C. Challioui, P. Mirebeau, and J. N. Demay, "Industrial applications of polyaniline," Synthetic Metals, vol. 101, no. 1, pp. 703-704, 1999.

[11] E. Smela, W. Lu, and B. R. Mattes, "Polyaniline actuators, part 1: PANI(AMPS) in HCl," Synthetic Metals, vol. 151, no. 1, pp. 25-42, 2005.

[12] P. Andersson, M. Berggren, and T. Kugler, "Switchable optical polarizer based on electrochromism in stretch-aligned polyaniline," Applied Physics Letters, vol. 83, no. 7, pp. 1307-1309, 2003.

[13] L. J. Shen, D. W. Gu, J. S. Li, H. Y. Zhou, W. R. Xiao, and N. R. Yang, "The growth of PANI films at $450 \mathrm{MPa}$," Synthetic Metals, vol. 155, no. 1, pp. 110-115, 2005.

[14] H. Karami, M. F. Mousavi, and M. Shamsipur, "A new design for dry polyaniline rechargeable batteries," Journal of Power Sources, vol. 117, no. 1-2, pp. 255-259, 2003.

[15] A. Rezzan and K. Faith, "Electrochemical reduction of $\mathrm{CO}_{2}$ on a polyaniline electrode under ambient conditions and at high pressure in methanol," Journal of Electroanalytical Chemistry, vol. 535, no. 1-2, pp. 107-112, 2002.

[16] D. W. Gu, J. S. Li, J. L. Liu, Y. M. Cai, and L. J. Shen, "PAN thin films in situ polymerized under very high pressure," Synthetic Metals, vol. 150, no. 2, pp. 175-179, 2005.

[17] S. J. Cui, D. P. Xu, W. H. Su, F. S. Wang, S. L. Wang, "Investigation on electrical properties of the PAN complexes under high pressure," Chinese Journal of High Pressure Physics, vol. 1, no. 1, p. 71, 1987.

[18] Z. X. Liu, D. L. Zhang, M. X. Wan, M. Li, J. C. Li, "Effect of high pressure on the conductivity of PAN," Chinese Journal of High Pressure Physics, vol. 9, no. 3, p. 183, 1995.

[19] W. S. Xiao, K. N. Weng, W. S. Peng, Z. X. Bao, C. X. Liu, X. R. Zeng, "Study of PAN FTIR and its resistance under high pressure," Chinese Journal of High Pressure Physics, vol. 11, no. 2, p. 84, 1997.

[20] Z. X. Bao, C. X. Liu, P. K. Kahol, and N. J. Pinto, "Pressure dependence of the resistance in polyaniline and its derivatives at room temperature," Synthetic Metals, vol. 106, no. 2, pp. 107-110, 1999.

[21] S. M. Hong, L. Y. Chen, X. R. Liu, X. H. Wu, and L. Su, "High pressure jump apparatus for measuring Gruneisen parameter of $\mathrm{NaCl}$ and studying metastable amorphous phase of poly (ethylene terephthalate)," Review of Scientific Instruments, vol. 76, no. 5, Article ID 053905, 2005.

[22] R. Hinrichs, A. Régis, A. Gruger, and P. Colomban, "Pressuretemperature-induced conductivity in polyaniline base and salts," Synthetic Metals, vol. 81, no. 2-3, pp. 227-231, 1996.

[23] A. Zherebov, A. Lachinov, V. Kornilov, and M. Zolotukhin, "Metal phase in electroactive polymer induced by uniaxial pressure," Synthetic Metals, vol. 84, no. 1-3, pp. 735-736, 1997.

[24] J. Huang and M. Wan, "Temperature and pressure dependence of conductivity of polyaniline synthesized by in situ doping polymerization in the presence of organic function acid as dopants," Solid State Communications, vol. 108, no. 4, pp. 255259, 1998.

[25] J. J. Langer, G. Framski, and R. Joachimiak, "Polyaniline nanowires and nano-networks," Synthetic Metals, vol. 121, no. 1-3, pp. 1281-1282, 2001.

[26] S. Stafström, J. L. Brédas, A. J. Epstein et al., "Polaron lattice in highly conducting polyaniline: theoretical and optical studies," Physical Review Letters, vol. 59, no. 13, pp. 1464-1467, 1987.

[27] S. Gokden, "Mobility of two-dimensional electrons in an AlGaN/GaN modulation-doped heterostructure," Physica Status Solidi A, vol. 200, no. 2, pp. 369-377, 2003. 

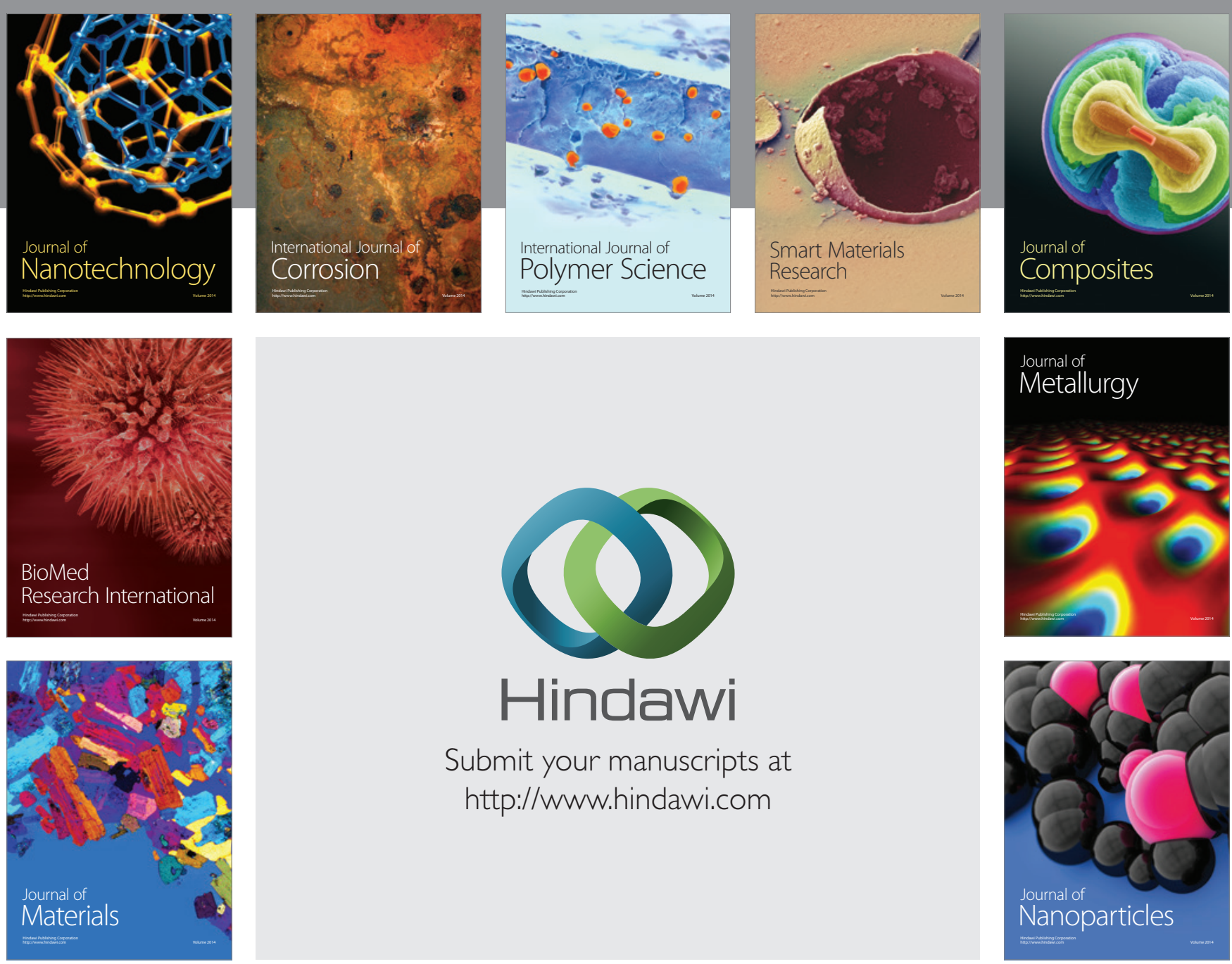

Submit your manuscripts at http://www.hindawi.com
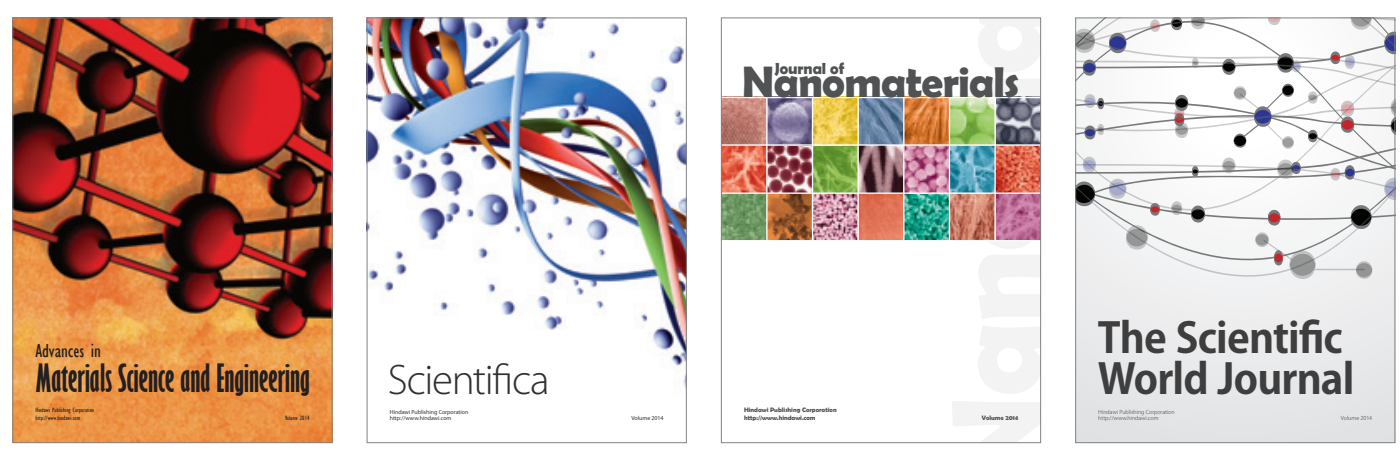

\section{The Scientific World Journal}
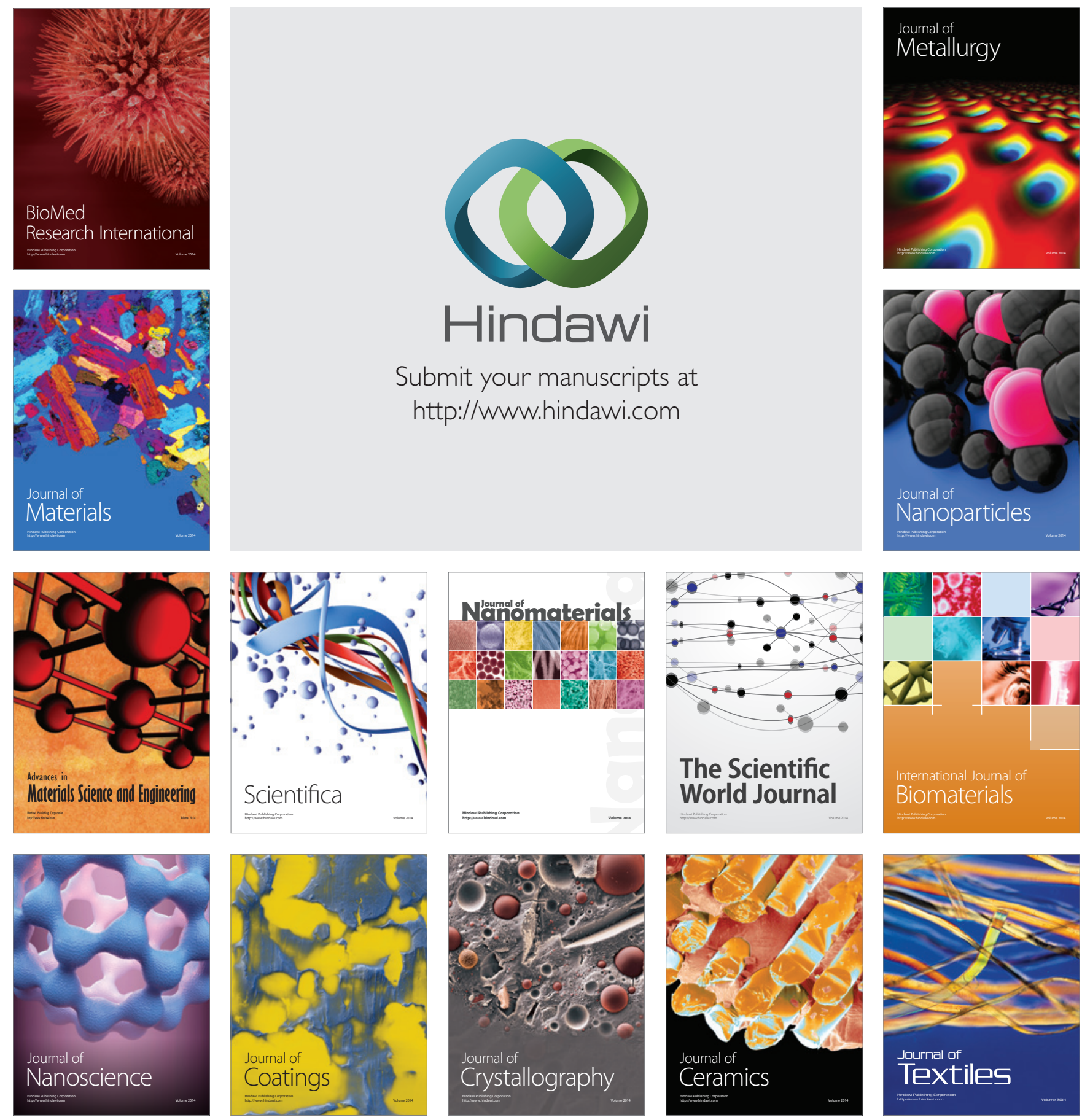